\title{
Heavy quark mass determination from the quarkonium ground state energy: a pole mass approach
}

\author{
Taekoon Lee* \\ Department of Physics, Seoul National University, Seoul 151-742, Korea and \\ National Center for Theoretical Sciences, \\ National Tsing Hua University, Hsinchu, Taiwan
}

\begin{abstract}
The heavy quark pole mass in perturbation theory suffers from a renormalon caused, inherent uncertainty of $O\left(\Lambda_{\mathrm{QCD}}\right)$. This fundamental difficulty of determining the pole mass to an accuracy better than the inherent uncertainty can be overcome by direct resummation of the first infrared renormalon. We show how a properly defined pole mass as well as the $\overline{\mathrm{MS}}$ mass for the top and bottom quarks can be determined accurately from the $O\left(m \alpha_{s}^{5}\right)$ quarkonium ground state energy.
\end{abstract}

*Electronic address: tlee@phya.snu.ac.kr 


\section{INTRODUCTION}

The pole mass is a central parameter in heavy quark physics but suffers from a renormalon caused ambiguity [1, 2]. In the heavy quark effective theory (HQET) it is defined formally as the heavy quark mass at which the residual mass term vanishes. This definition of the pole mass is however incomplete. In general, the parameters and the coefficient functions of HQET should be related to the fundamental quantum chromodynamics (QCD) Lagrangian through the matching process. In particular, in perturbation theory the pole mass is related to the high energy mass such as the $\overline{\mathrm{MS}}$ mass $m_{\overline{\mathrm{MS}}}\left(\equiv m_{\overline{\mathrm{MS}}}\left(m_{\overline{\mathrm{MS}}}\right)\right)$ by

$$
m_{\text {pole }}=m_{\overline{\mathrm{MS}}}\left(1+\sum_{n=0}^{\infty} p_{n} \bar{\alpha}_{s}^{n+1}\right),
$$

where $\bar{\alpha}_{s}=\alpha_{s}(m \overline{\mathrm{MS}})$ denotes the strong coupling. The problem with this expansion is that it is an asymptotic expansion, with a large order behavior [3],

$$
p_{n}=C_{m} \frac{(n+\nu) !}{\nu !}\left(2 \beta_{0}\right)^{n}[1+O(1 / n)]
$$

where $C_{m}$ is a normalization constant and $\nu=\beta_{1} / 2 \beta_{0}^{2}$, with $\beta_{0}, \beta_{1}$ denoting the first two coefficients of the QCD beta function. Because of the factorial divergence the expansion (11) contains an intrinsic uncertainty of order

$$
m_{\overline{\mathrm{MS}}} \exp \left(-\frac{1}{2 \beta_{0} \bar{\alpha}_{s}}\right) \propto \Lambda_{\mathrm{QCD}},
$$

which means that within the perturbation theory the pole mass cannot be determined to an accuracy better than $O\left(\Lambda_{\mathrm{QCD}}\right)$ even if the $\overline{\mathrm{MS}}$ mass is known.

On the other hand, one may try to extract the pole mass from the predictions of physical observables in HQET. But, as well known, this too does not work since the predictions of HQET as well suffer from the renormalon caused uncertainty. As an example, consider the inclusive semileptonic $B$ decay rate in HQET,

$$
\Gamma\left(B \rightarrow X_{u e \bar{\nu}_{e}}\right)=\frac{G_{\mathrm{F}}^{2}\left|V_{\mathrm{ub}}\right|^{2}}{192 \pi^{3}}\left(m_{\text {pole }}^{(b)}\right)^{5} f\left(\alpha_{s}\right)\left\{1+O\left[1 /\left(m_{\text {pole }}^{(b)}\right)^{2}\right]\right\} .
$$

$f\left(\alpha_{s}\right)$ contains the QCD corrections to the free $b$ quark decay and can be expanded in power series of the strong coupling. This expansion also suffers from the factorially growing divergence similar to that in Eq. (2) and has an intrinsic uncertainty of $O\left(\Lambda_{\mathrm{QCD}} / m_{\text {pole }}^{(b)}\right)$ [1, 4]. Because of this uncertainty the bottom quark pole mass $m_{\text {pole }}^{(b)}$ cannot be extracted to an accuracy better than $O\left(\Lambda_{\mathrm{QCD}}\right)$, even assuming the decay rate is measured precisely and the Cabibbo-Kobayashi-Maskawa matrix element $V_{\mathrm{ub}}$ is known.

Formally, this problem can be resolved by Borel resummation of the divergent series. Consider again the pole mass expansion (11). The resummed pole mass can be written as

$$
m_{\text {pole }}=m_{\mathrm{BR}} \pm i \Gamma_{m}
$$

where $m_{\mathrm{BR}}$ and $\Gamma_{m}$ denote the real and the imaginary parts of the Borel integral, respectively. The imaginary part arises from the renormalon singularity that gives rise to the large order 
behavior (2), and is ambiguous; its sign depends on the integration contour taken. In general, an unphysical, ambiguous imaginary part in Borel resummation implies presence of nonperturbative effect and is supposed to be canceled by the latter. In the case of the pole mass, however, the ambiguity is spurious in that in physical observables it is canceled by a corresponding ambiguity in another divergent series in the coefficient functions or the matrix elements $[4,5,6]$. For example, in the $B$ decay rate the ambiguous imaginary part in the resummed pole mass is canceled by the ambiguity in the resummed $f$. Thus, as far as physical observables are concerned, the introduction of the nonperturbative effect is not necessary.

Since the ambiguous imaginary parts in Borel resummations in HQET are canceled in physical observables we can entirely ignore them and keep only the real parts, the 'BR' quantities. Under this 'BR' prescription of Borel resummation the pole mass is defined as

$$
m_{\text {pole }} \equiv m_{\mathrm{BR}}
$$

Throughout the paper we take this as the definition of the pole mass, unless implied otherwise. By definition the BR mass, when expanded in the strong coupling constant, has exactly the same perturbative coefficients as the pole mass in perturbation theory.

Although this formal argument resolves the renormalon problem the actual computation of the 'BR' quantities is another matter. Since the ambiguity in Borel resummation arises from the renormalon singularity, to calculate a BR quantity to an accuracy better than $O\left(\Lambda_{\mathrm{QCD}}\right)$ it is necessary to have a precise description of the Borel transform in the region that includes the origin and the renormalon singularity. Within the perturbation theory only the first few terms of the power expansion of the Borel transform about the origin are known, so describing the Borel transform beyond the immediate neighborhood of the origin can be a difficult task, since it would require a precise knowledge on the large order behavior.

Describing the Borel transform accurately about the renormalon singularity is equivalent to having an accurate information on the associated large order behavior. Since the essential information in the large order behavior that is missing is the normalization constant (residue) its computation will make the computation of BR quantity possible. Fortunately, the normalization constant can be calculated in perturbation theory [7, 8], and the residue of the pole mass expansion can be computed accurately, within a few percent error, from the known next-next-leading order (NNLO) calculations of the expansion (11) [9, 10, 11]. This accurate computation of the pole mass residue is important, because the residues of other divergent series whose leading IR renormalon is cancelled by that of the pole mass can be obtained to the same accuracy.

Once the residue is known the Borel transform can be expanded systematically about the renormalon singularity. Then by interpolating this expansion with the perturbative expansion about the origin we can obtain an accurate description of the Borel transform in the region that contains the origin and the singularity. The interpolation itself is encoded in what we call bilocal expansion.

This idea of bilocal expansion of the Borel transform was successfully applied to the heavy quark static potential and the inclusive semileptonic $B$ decay [9, 10]. The Borel resummed heavy quark potential, for example, at short and intermediate distances agrees remarkably well with lattice calculations.

So far, the renormalon problem in heavy quark physics was handled essentially by avoiding it by only considering physical observables. Since physical observables are free from renormalons, one can avoid the renormalon problem by replacing the pole mass with a 
renormalon-free (short distance) mass. Instead, the bilocal expansion allows us to confront the renormalon problem directly, and render us infrared sensitive quantities at our disposal.

In this paper we apply this technique to the perturbative expansion of the heavy quarkonium ground state energy. Our purpose is two fold. First we aim to demonstrate that the scheme described above indeed allows us to extract a properly defined pole mass accurately from experimental data. It is often claimed that a pole mass cannot be determined to accuracy better than $O\left(\Lambda_{\mathrm{OCD}}\right)$. We provide here a counterproof. Secondly, we aim to re-examine the analysis in Ref. [12] which extracts from the quarkonium binding energy the top and bottom quark masses in the pole mass scheme but without taking into account the renormalon effect. We show that a proper handling of the renormalon results in sizable shifts in the extracted quark masses.

\section{THE METHOD}

In this section we give a brief summary of the resummation method of the first infrared (IR) renormalon in heavy quark physics using the bilocal expansion. For details we refer the reader to 9, 10

Assume a physical quantity $A\left(\alpha_{s}\right)$ has perturbative expansion

$$
A\left(\alpha_{s}\right)=\sum_{n=0}^{\infty} a_{n} \alpha_{s}^{n+1}
$$

and a renormalon ambiguity proportional to $\Lambda_{\mathrm{QCD}}$. Then the Borel transform $\tilde{A}(b)$ defined by the Borel integral,

$$
A\left(\alpha_{s}\right)=\frac{1}{\beta_{0}} \int_{0 \pm i \epsilon}^{\infty \pm i \epsilon} e^{-b / \beta_{0} \alpha_{s}} \tilde{A}(b) d b
$$

has the perturbative expansion about the origin,

$$
\tilde{A}(b)=\sum_{n=0}^{\infty} \frac{a_{n}}{n !}\left(\frac{b}{\beta_{0}}\right)^{n},
$$

and about the renormalon singularity at $b=1 / 2$ of the form

$$
\tilde{A}(b)=\frac{C}{(1-2 b)^{1+\nu}}\left(1+c_{1}(1-2 b)+c_{2}(1-2 b)^{2}+\cdots\right)+(\text { analytic part }),
$$

where $C$ and $c_{i}$ are real constants and $\nu$ same as in (2). The coefficients $c_{i}$ can be determined by expanding $\Lambda_{\mathrm{QCD}}$ in $\alpha_{s}$, and they depend only on the beta function coefficients. In terms of the known four loop beta function the first two coefficients can be found as [3]

$$
c_{1}=\frac{\beta_{1}^{2}-\beta_{0} \beta_{2}}{4 \nu \beta_{0}^{4}}, \quad c_{2}=\frac{\beta_{1}^{4}+4 \beta_{0}^{3} \beta_{1} \beta_{2}-2 \beta_{0} \beta_{1}^{2} \beta_{2}+\beta_{0}^{2}\left(\beta_{2}^{2}-2 \beta_{1}^{3}\right)-2 \beta_{3} \beta_{0}^{4}}{32 \nu(\nu-1) \beta_{0}^{8}} .
$$

The "analytic part" in (10) denotes terms analytic on the disk $|b-1 / 2|<1$ about the singularity. The radius of convergence of the expansion (9) is expected to be bounded by the first IR renormalon at $b=1 / 2$ and that of the expansion (10) bounded by the second renormalon at $b=3 / 2$. The Borel transforms of the pole mass and the quarkonium binding energy are expected to satisfy all these conditions. 
As noted above, to compute the Borel integral (8) to an accuracy better than $O\left(e^{-1 / 2 \beta_{0} \alpha_{s}}\right)$ we must have an accurate description of the Borel transform in the region that includes both the origin and the first IR renormalon singularity. This can be done with the bilocal expansion that interpolates the two expansions (9) and (10),

$$
\tilde{A}(b)=\lim _{N, M \rightarrow \infty} \tilde{A}_{\mathrm{N}, \mathrm{M}}(b) \text {, }
$$

where

$$
\tilde{A}_{\mathrm{N}, \mathrm{M}}(b)=\sum_{n=0}^{N} \frac{h_{n}}{n !}\left(\frac{b}{\beta_{0}}\right)^{n}+\frac{C}{(1-2 b)^{1+\nu}}\left[1+\sum_{i=1}^{M} c_{i}(1-2 b)^{i}\right] .
$$

The coefficients $h_{n}$ are to be determined by demanding the bilocal expansion have the same perturbative expansion about the origin as (9). The first two coefficients $c_{1,2}$ are known, so taking $M=2$ in (13) we then have

$$
\begin{aligned}
& h_{0}=a_{0}-C\left(1+c_{1}+c_{2}\right), \\
& h_{1}=a_{1}-2 C \beta_{0}\left[1-c_{2}+\nu\left(1+c_{1}+c_{2}\right)\right], \\
& h_{2}=a_{2}-4 C \beta_{0}^{2}\left[2+\nu\left(3+c_{1}-c_{2}\right)+\nu^{2}\left(1+c_{1}+c_{2}\right)\right], \\
& h_{3}=a_{3}-8 C \beta_{0}^{3}(1+\nu)\left[6+\nu\left(5+2 c_{1}-c_{2}\right)+\nu^{2}\left(1+c_{1}+c_{2}\right)\right], \text { etc. }
\end{aligned}
$$

The interpolating Borel transform $\tilde{A}_{\mathrm{N}, \mathrm{M}}(b)$ implements the correct nature of the first renormalon singularity and this allows us to resum the renormalon caused large order behavior to all orders. A quick comparison between (10) and (13) shows that the expansion about the origin in (13), those with the coefficients $h_{n}$, simulates the "analytic part" in (10).

Clearly, a working bilocal expansion requires computation of the renormalon residue. The residue can be calculated perturbatively in a straightforward manner following the observation in [7, 8]. To compute the residue, notice that

$$
C=R\left(\frac{1}{2}\right)
$$

where

$$
R(b) \equiv(1-2 b)^{1+\nu} \tilde{A}(b) .
$$

Now the value of the function $R(b)$ at $b=\frac{1}{2}$ can be evaluated by a series expansion in powers of $\frac{1}{2}$, since $R(b)$ is expected to be analytic on the disk $|b|<1 / 2$ and is bounded at $b=1 / 2$. Even though the evaluation occurs precisely on the boundary of the convergence disk, the boundedness of $R$ on the boundary guarantees convergence. Note that for the series expansion of $R(b)$ only the Borel transform in perturbative form (9) is needed.

The convergence of the power expansion for the residue can be improved by rendering the function $R$ to be smoother on the disk $|b|<1 / 2$. This can be done by pushing away from the origin the ultraviolet renormalons and the subleading IR renormalons through an employment of a conformal mapping. For the case of the pole mass and the quarkonium energy we can use the mapping:

$$
w=\frac{\sqrt{1+b}-\sqrt{1-2 b / 3}}{\sqrt{1+b}+\sqrt{1-2 b / 3}}
$$

which maps the first IR renormalon to $w=1 / 5$ and all others onto the unit circle. In $w$ plane the residue can be obtained by expanding $R[b(w)]$ about the origin in power series, and evaluating it at $w=1 / 5$. 


\section{RELATION BETWEEN THE POLE MASS AND THE $\overline{\mathrm{MS}}$ MASS}

In this section we show that the relation between the pole mass and the $\overline{\mathrm{MS}}$ mass in the case of the bottom and top quark can be given to an accuracy much better than $O\left(\Lambda_{\mathrm{QCD}}\right)$. Writing the expansion (II) as

$$
m_{\text {pole }}=m_{\overline{\mathrm{MS}}}\left[1+\mathcal{M}\left(\bar{\alpha}_{s}\right)\right], \quad \mathcal{M}\left(\bar{\alpha}_{s}\right)=\sum_{n=0}^{\infty} p_{n} \bar{\alpha}_{s}^{n+1}
$$

the Borel transform $\tilde{\mathcal{M}}(b)$ of $\mathcal{M}\left(\bar{\alpha}_{s}\right)$ has the first IR renormalon singularity precisely of the form (10).

To employ the bilocal expansion for the summation of the series for $\mathcal{M}$ we first need the residue $C_{m}$ for the pole mass, corresponding to $C$ in Eq. (10). Following the description in the previous section and using the known first three coefficients 13, 14, 15]

$$
\begin{aligned}
& p_{0}=0.4244 \\
& p_{1}=1.3621-0.1055 n_{f}, \\
& p_{2}=6.1404-0.8597 n_{f}+0.0211 n_{f}^{2},
\end{aligned}
$$

where $n_{f}$ denotes the number of light quark flavors, we obtain the residue

$$
C_{m}=\left\{\begin{array}{l}
0.4244+0.1151-0.0099 \pm 0.0080=0.5296 \pm 0.0080,\left(n_{f}=5\right) \\
0.4244+0.1224+0.0101 \pm 0.0080=0.5569 \pm 0.0080,\left(n_{f}=4\right)
\end{array} .\right.
$$

The errors were obtained by taking the differences between the residues evaluated at NNLO and at next-next-next-leading order (NNNLO), with the latter computed using the NNNLO coefficients estimated following the method in [16]. The good convergence of the residue at such a low order perturbation is indeed remarkable, and it can be traced to the renormalon dominance in the perturbation expansion of the pole mass.

Using the values for $C_{m}$ above and the coefficients (19) we can obtain the interpolating Borel transforms $\tilde{\mathcal{M}}_{0,2}(b), \tilde{\mathcal{M}}_{1,2}(b)$, and $\tilde{\mathcal{M}}_{2,2}(b)$. With these Borel transforms it is now easy to do the actual Borel summation. The integration in the Borel integral can be performed easily in $w$ plane, defined by (17), with the contour now along a ray off the origin to the unit circle in the first (or fourth) quadrant [17]. Taking the real part of the integral we have

$m_{\mathrm{BR}}=\left\{\begin{array}{ll}m_{\overline{\mathrm{MS}}}(1+0.060336+0.001310-0.000029 \pm 0.000046), & \left(n_{f}=5, \text { top quark }\right) \\ m_{\overline{\mathrm{MS}}}(1+0.1577+0.0041-0.0003 \pm 0.0001), & \left(n_{f}=4, \text { bottom quark }\right)\end{array}\right.$.

In this computation we have taken, for demonstration purpose, $\alpha_{s}^{[5]}\left(m_{\overline{\mathrm{MS}}}\right)=0.108$ and $\alpha_{s}^{[4]}\left(m_{\overline{\mathrm{MS}}}\right)=0.22$ for the top and bottom quarks, respectively. The errors are from the uncertainties in the computed residues in (20). Notice the remarkable convergence compared to the unresummed series at the same $\alpha_{s}$,

$$
m_{\text {pole }}=\left\{\begin{array}{ll}
m_{\overline{\mathrm{MS}}}(1+0.0458+0.0097+0.0030), & \left(n_{f}=5, \text { top quark }\right) \\
m_{\overline{\mathrm{MS}}}(1+0.093+0.045+0.032), & \left(n_{f}=4, \text { bottom quark }\right)
\end{array} .\right.
$$

Taking $m_{\overline{\mathrm{MS}}}=165 \mathrm{GeV}$ for the top and $m_{\overline{\mathrm{MS}}}=4.2 \mathrm{GeV}$ for the bottom quark we see the uncertainties in the resummed relations are less than $10 \mathrm{MeV}$ and $1 \mathrm{MeV}$, respectively much smaller than $\Lambda_{\overline{\mathrm{MS}}}$. 


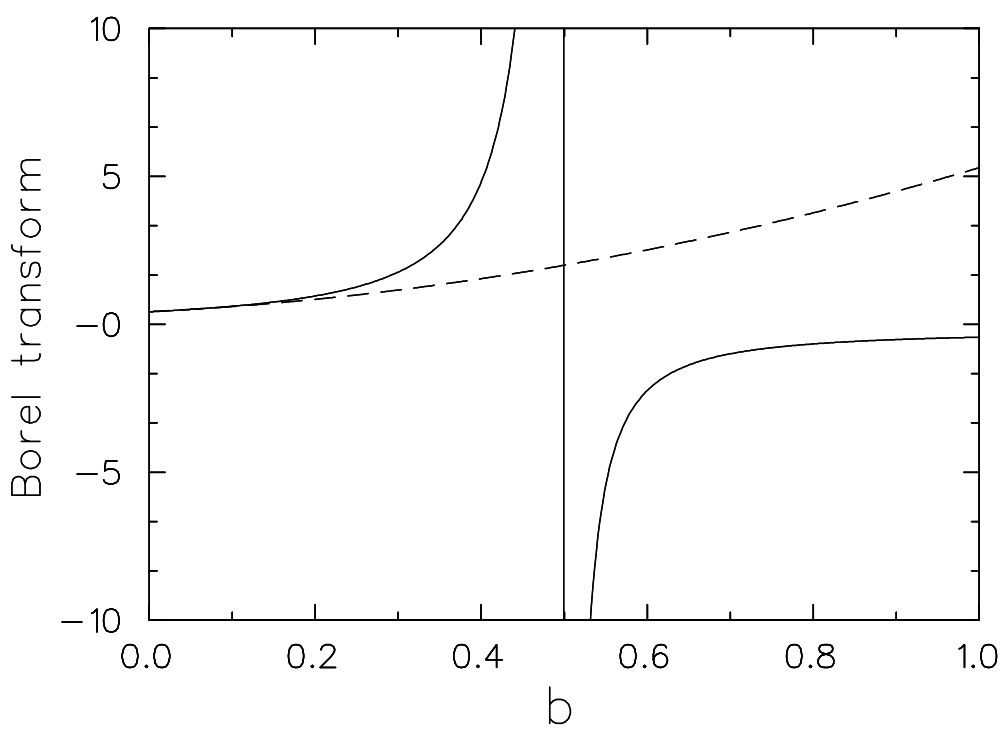

FIG. 1: Interpolating Borel transform $\tilde{\mathcal{M}}_{2,2}(b)$ (solid) vs ordinary NNLO perturbative Borel transform (dashed).

The drastic improvement in the convergence comes from the proper treatment of the renormalon singularity in the Borel transform. For comparison, we plot in Fig. 11 the ordinary NNLO Borel transform in power series against the real part of the interpolating Borel transform $\tilde{\mathcal{M}}_{2,2}(b)$ at $n_{f}=4$. Though they agree on the domain about the origin, as should, the deviation becomes obvious at larger values of $b$, close and beyond the singularity at $b=1 / 2$.

The calculation so far clearly shows that direct resummation of the first infrared renormalon is not only feasible but can be efficient.

\section{RESUMMATION OF THE BINDING ENERGY}

Having established a precise connection between the pole mass and the $\overline{\mathrm{MS}}$ mass we proceed to the resummation of the binding energy of the quarkonium $1 S$ state. Since we have nothing new to add to the nonperturbative effects or the heavy quark finite width effect we shall assume the quarks are stable and the system is completely perturbative. Those effects may be added later on to the perturbative result.

Since the early works by Titard and Yndurain [18, 19] there has been many studies on the precision calculation of the quarkonium energy within perturbative QCD. The binding energy is known to NNLO, and the partial computations of NNNLO energy are also incorporated in recent works [11, 12, 20, 21].

The quarkonium $1 S$ energy $M_{1 S}$ is given as the sum of the pole mass and the binding energy $E$,

$$
\begin{aligned}
M_{1 S} & =2 m_{\mathrm{pole}}+E \\
& =2 m_{\mathrm{BR}}+E_{\mathrm{BR}} .
\end{aligned}
$$


In perturbation theory the binding energy can be expanded as

$$
E=-\bar{\mu}(\mu) \sum_{n=0}^{\infty} \mathcal{E}_{n}(\xi) \alpha_{s}(\mu)^{n+1},
$$

where $\mu$ is the renormalization scale and

$$
\bar{\mu}(\mu)=C_{F} \alpha_{s}(\mu) m_{\mathrm{BR}}, \quad \xi \equiv \frac{\mu}{\bar{\mu}},
$$

and $C_{F}=4 / 3$. The first three coefficients are known [18, 22], and, for $m_{\mathrm{BR}} \alpha_{s}^{2} \gg \Lambda_{\mathrm{QCD}}$, the fourth almost [12, 23, 24, 25], with only the three loop contribution to the static potential not calculated. They are given by $\mathcal{E}_{n}=\frac{1}{3} P_{n}$ with

$$
\begin{aligned}
P_{0}= & 1, \quad P_{1}=4 \beta_{0} L+k_{1}, \\
P_{2}= & 12 \beta_{0}^{2} L^{2}+\left(-8 \beta_{0}^{2}+4 \beta_{1}+6 \beta_{0} k_{1}\right) L+k_{2}, \\
P_{3}= & 32 \beta_{0}^{3} L^{3}+\left(-56 \beta_{0}^{3}+28 \beta_{0} \beta_{1}+24 \beta_{0}^{2} k_{1}\right) L^{2} \\
& +\left(16 \beta_{0}^{3}-16 \beta_{0} \beta_{1}+4 \beta_{2}-12 \beta_{0}^{2} k_{1}+6 \beta_{1} k_{1}+8 \beta_{0} k_{2}\right) L+k_{3},
\end{aligned}
$$

where $L=\ln (\xi)$ and

$$
\begin{aligned}
k_{1}= & \frac{1}{\pi}\left(97 / 6-11 n_{f} / 9\right) \\
k_{2}= & \frac{1}{\pi^{2}}\left(337.9471-40.96485 n_{f}+1.162857 n_{f}^{2}\right), \\
k_{3}= & \frac{1}{\pi^{3}}\left(7078.7900-1215.5475 n_{f}+69.450816 n_{f}^{2}-1.21475 n_{f}^{3}\right. \\
& \left.+0.031250 a_{3}+474.2893 \ln \left[\alpha_{s}(\mu)\right]\right),
\end{aligned}
$$

and the beta function coefficients,

$$
\begin{aligned}
& \beta_{0}=\frac{1}{4 \pi}\left(11-2 n_{f} / 3\right), \quad \beta_{1}=\frac{1}{(4 \pi)^{2}}\left(102-38 n_{f} / 3\right) \\
& \beta_{2}=\frac{1}{(4 \pi)^{3}}\left(2857 / 2-5033 n_{f} / 18+325 n_{f}^{2} / 54\right) .
\end{aligned}
$$

$a_{3}$ in (27) denotes the unknown three loop coefficient in the $\overline{\mathrm{MS}}$ scheme of the static potential in momentum space. Using the Borel transform method of estimating unknown higher order coefficients [16], we can find an estimate of the three loop coefficient $V_{3}$ of the static potential in coordinates space,

$$
V(r)=\frac{1}{r} \sum_{n=0}^{\infty} V_{n} \alpha_{s}(1 / r)^{n+1},
$$

which reads

$$
V_{3}= \begin{cases}-19.33 \pm 2.73, & \left(n_{f}=5\right) \\ -27.03 \pm 3.50, & \left(n_{f}=4\right)\end{cases}
$$

The corresponding $a_{3}$ is then given by ${ }^{1}$

$$
\frac{a_{3}}{4^{3}}=\left\{\begin{array}{ll}
34 \mp 63, & \left(n_{f}=5\right) \\
59 \mp 81, & \left(n_{f}=4\right)
\end{array} .\right.
$$

\footnotetext{
${ }^{1}$ This agrees with the existing estimates within the errors, $a^{3} / 4^{3}=60,98$ from the Padè [26] and $a^{3} / 4^{3}=$ 37,72 from the large order behavior [11], for $n_{f}=5,4$, respectively.
} 
Note the errors in $V_{3}$ estimates get amplified in the conversion from $V_{3}$ to $a_{3}$ by an order of magnitude, resulting in large errors in the latter. So a few $100 \%$ error in $a_{3}$ should be counted as normal.

The binding energy has a renormalon caused ambiguity proportional to $\Lambda_{\mathrm{QCD}}$ which is to be canceled by that of the pole mass term $\left(2 m_{\text {pole }}\right)$ [27, 28, 29], and so the Borel transform $\tilde{E}$ of the binding energy can be expanded about the renormalon singularity as

$$
\tilde{E}(b, \xi)=-\frac{2 C_{m} \xi \bar{\mu}}{(1-2 b)^{1+\nu}}\left(1+c_{1}(1-2 b)+c_{2}(1-2 b)^{2}+\cdots\right)+(\text { analytic part })
$$

with $C_{m}$ and $c_{1,2}$ given by (20) and (11), respectively. Note that in Eq. (32) the renormalon residue of $\tilde{E}$ is expressed in terms of the pole mass residue $C_{m}$, using the renormalon cancellation between the pole mass and the binding energy. This is to utilize the accurate computation of the pole mass residue. One can, of course, proceed without refereeing to it, by directly computing the residue from the coefficients (26); however, the convergence is not as good as in the pole mass residue [9, 10, 11]. Combining (32) with the expansion about the origin,

$$
\tilde{E}(b, \xi)=-\bar{\mu} \sum_{n=0}^{\infty} \frac{\mathcal{E}_{n}(\xi)}{n !}\left(\frac{b}{\beta_{0}}\right)^{n},
$$

into a bilocal expansion following the description in Sec. II we can obtain, using the known coefficients (26) , the first four interpolating Borel transforms $\tilde{E}_{N, 2}(b, \xi)(N=0,1,2,3)$.

The Borel resummed binding energy $E_{\mathrm{BR}}$ can then be obtained by substituting $\tilde{E}(b, \xi)$ with $\tilde{E}_{N, 2}(b, \xi)$ in the integral

$$
E_{\mathrm{BR}}=\operatorname{Re}\left[\frac{1}{\beta_{0}} \int_{0 \pm i \epsilon}^{\infty \pm i \epsilon} e^{-b / \beta_{0} \alpha_{s}(\mu)} \tilde{E}(b, \xi) d b\right]
$$

In the following we take $\alpha_{s}^{[5]}\left(M_{Z}\right)=0.1172 \pm 0.002$ [30], and use the four loop beta function for the computation of the running coupling $\alpha_{s}(\mu)$.

\section{A. Top quark}

Because of the large mass and decay width of the top quark the toponium $1 S$ state is expected to have small nonperturbative effects and to be well described by perturbation theory. In future $e^{+} e^{-}$linear colliders the toponium energy $M_{1 S}$ is expected to be measured precisely from the peak position of top threshold production cross section. This provides a unique opportunity for precision determination of the top mass.

We now perform the Borel integration in (34) with the interpolating Borel transforms. The results are plotted in Fig. 2 at the pole mass value $m_{\mathrm{BR}}=175 \mathrm{GeV}$.

Notice the very small renormalization scale dependence in the NNNLO resummed energy. It is less than $10 \mathrm{MeV}$ over the range $30 \mathrm{GeV} \leq \mu \leq 150 \mathrm{GeV}$ with $a_{3} / 4^{3}=34$. For comparison we plot the unresummed binding energies in Fig. 3] using the same pole mass. Compared to the unresummed binding energies the improvement of the resummed is clear. They have much smaller perturbation order dependence and scale dependence. It is evident that for precise determination of the binding energy the renormalon must be taken into account properly. 


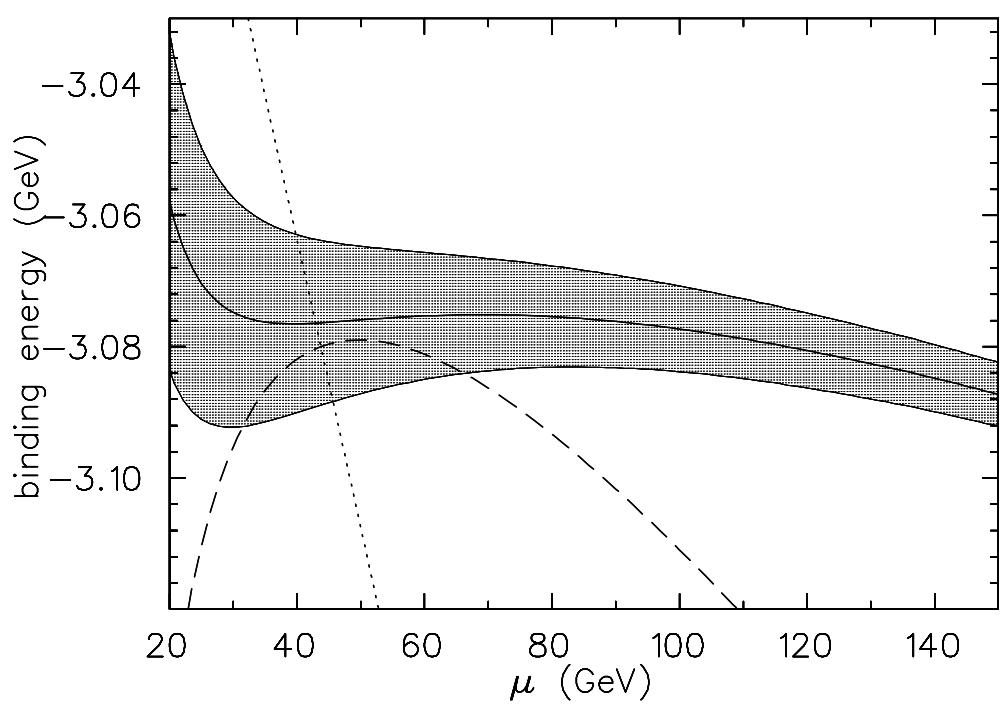

FIG. 2: The resummed toponium $1 S$ state binding energy using $\tilde{E}_{1,2}$ (dotted), $\tilde{E}_{2,2}$ (dashed), and $\tilde{E}_{3,2}$ (solid). The shaded band denotes the variation due to the uncertainty in the estimate of $a_{3}$ in Eq. (31). The central line, upper, and lower boundaries correspond to $a_{3} / 4^{3}=34,-29$, and 97 , respectively.

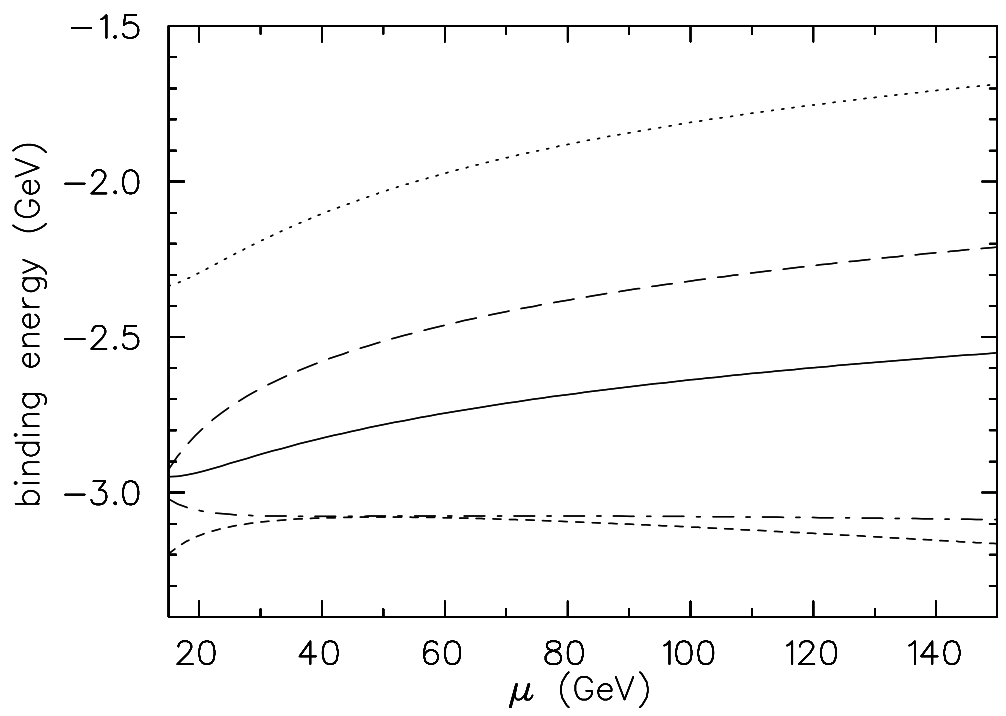

FIG. 3: The unresummed toponium $1 S$ state binding energy at NLO(dotted), NNLO(dashed), and NNNLO (solid). For comparison the resummed binding energies using $\tilde{E}_{2,2}$ (short-dashed) and $\tilde{E}_{3,2}$ (dot-dashed) are given. The same pole mass was employed in the resummed and unresummed cases.

The dominant theoretical uncertainty in the resummed binding energy comes from the uncertainty in the strong coupling constant. In the pole mass scheme under consideration there is a natural scale for the binding energy, the Bohr scale $\bar{\mu}\left(\mu_{\text {Bohr }}\right)=\mu_{\text {Bohr }}$, with $\mu_{\text {Bohr }} \approx$ $33 \mathrm{GeV}$. We expect an optimal result would come around this scale. Thus, taking the variation of the binding energy at the Bohr scale under $\delta \alpha_{s}^{[5]}\left(M_{Z}\right)=0.002$ we estimate the error caused by the strong coupling to be about $\pm 150 \mathrm{MeV}$ to the binding energy, which 


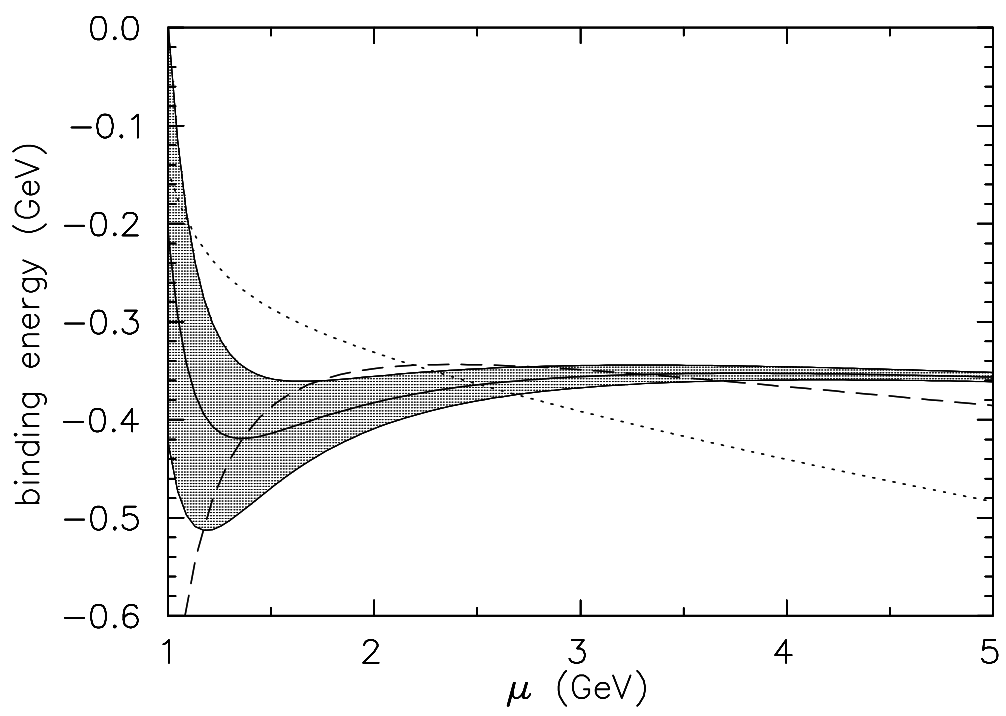

FIG. 4: The resummed $\Upsilon(1 S)$ binding energy using $\tilde{E}_{1,2}$ (dotted), $\tilde{E}_{2,2}$ (dashed), and $\tilde{E}_{3,2}$ (solid). The shaded band denotes the variation due to the uncertainty in the estimate of $a_{3}$ in Eq. (31). The central line, upper and lower boundaries correspond to $a_{3} / 4^{3}=59,-22$, and 140 , respectively.

would give $\pm 75 \mathrm{MeV}$ uncertainty in the pole mass. Other than the strong coupling the main source of uncertainty is $a_{3}$, as can be seen in Fig. 2. Comparatively, the renormalization scale dependence is small. In Fig. 2 we see that the binding energies at next-leading order (NLO), NNLO and NNNLO come close together in the range $\mu_{\mathrm{Bohr}} \leq \mu \leq 80 \mathrm{GeV}$, and also the principle of minimal sensitivity (PMS) 31] scales for the NNLO and NNNLO curves lie within the same interval. The renormalization scale dependence over this interval of the central NNNLO line is negligible, less than $2 \mathrm{MeV}$. The uncertainty in the renormalon residue at $n_{f}=5$ in (20) causes $\pm 5 \mathrm{MeV}$ error to the binding energy. The uncertainty in $a_{3}$ causes another $\pm 16 \mathrm{MeV}$ error in the binding energy. Combining these errors we conclude that the current theoretical error, excluding that caused by the strong coupling constant, on the pole mass is $\pm 10 \mathrm{MeV}$.

Once the pole mass is extracted from the binding energy the top mass in the $\overline{\mathrm{MS}}$ scheme can be determined using the relation between the BR mass and $\overline{\mathrm{MS}}$ mass discussed in Sec. III. The conversion introduces another error due to the uncertainty in the relation, about \pm 7 $\mathrm{MeV}$ to the $\overline{\mathrm{MS}}$ top mass. Combining these uncertainties in quadrature the corresponding theoretical uncertainty in the $\overline{\mathrm{MS}}$ mass is about $\pm 12 \mathrm{MeV}$.

\section{B. bottom quark}

The resummation of the $\Upsilon(1 S)$ binding energy can proceed in the same way as in the toponium, only with a few changes of the parameters. We take the renormalon residue and

$a_{3}$ estimates in (20) and (31), respectively, for $n_{f}=4$, and for the strong coupling $\alpha_{s}^{[4]}(\mu)$ we use RunDec [32] with the four loop beta function and three loop matching.

The resummed binding energies from the interpolating Borel transforms at NLO, NNLO, and NNNLO are plotted in Fig. 4 at the pole mass $m_{\mathrm{BR}}=4.9 \mathrm{GeV}$. For comparison the unresummed binding energies are plotted in Fig. [5. Again, the improvement of the resummed energy is clear. It has better convergence and less scale dependence. 


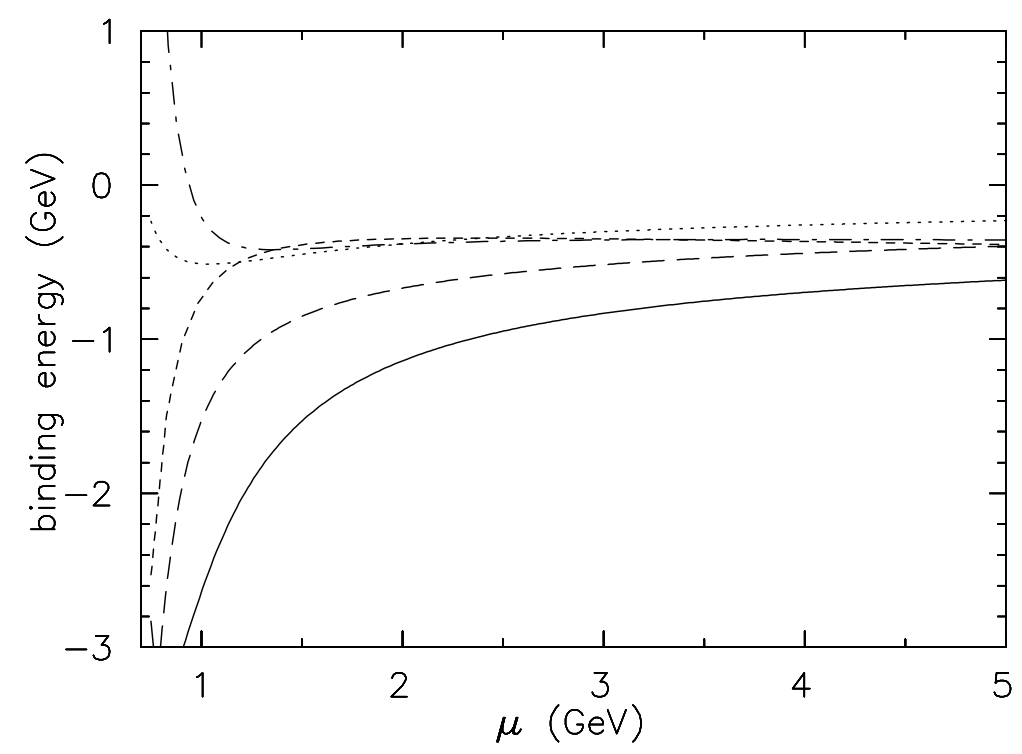

FIG. 5: The unresummed $\Upsilon(1 S)$ binding energy at NLO(dotted), NNLO(dashed), and NNNLO (solid). For comparison the resummed binding energies using $\tilde{E}_{2,2}$ (short-dashed) and $\tilde{E}_{3,2}$ (dotdashed) are given. The same pole mass was employed in the resummed and unresummed cases.

Before the BR mass can be extracted from the fitting of the resummed energy to the precisely measured $\Upsilon(1 S)$ mass, the nonperturbative effects and the charm mass effect should be subtracted from the $\Upsilon(1 S)$ mass. Without taking these effects into account we obtain $m_{\mathrm{BR}} \approx 4.9 \mathrm{GeV}$. The leading nonperturbative effect comes from the Stark effect of the long range gluon fields in the QCD vacuum [33, 34]. When the correlation time of the quarkonium state is much smaller than $1 / \Lambda_{\mathrm{QCD}}$ the nonperturbative effect may be expressed in terms of local gluon condensate. Whether $\Upsilon(1 S)$ is such a state is not clear, but assuming so, the nonperturbative effect is given by

$$
\frac{1872}{1275} \frac{m_{\mathrm{BR}} \pi<\alpha_{s} G_{\mu \nu}^{2}>}{\left(C_{F} \alpha_{s} m_{\mathrm{BR}}\right)^{4}} .
$$

The gluon condensate is poorly known. In general, fitted power corrections are contaminated by perturbative corrections, and sensitive to the perturbation order employed in fitting. For gluon condensate, it has been observed [35] that with higher order perturbation the value extracted is much smaller than the original sum rule value in [36]. Since in the binding energy the perturbative correction is already at relatively high order (NNNLO) ${ }^{2}$ the condensate for Eq. (35) should not contain significant perturbative corrections, and thus likely to be smaller than the original sum rule value. Here we take $<\alpha_{s} G_{\mu \nu}^{2}>=0.02 \pm 0.02$. Since the local condensate approximation for the nonperturbative effect is likely to provide an upper bound for any potential nonlocal effect [38], it may be reasonable to assume that this value covers adequately the nonperturbative effect. With $m_{\mathrm{BR}}=4.9 \mathrm{GeV}$ we then get the nonperturbative effect $60 \pm 60 \mathrm{MeV}$ in the binding energy. For the nonzero charm mass effect we find, using the result in [39, 40], $-20 \pm 15 \mathrm{MeV}$ in the binding energy. Subtracting these two effects from the experimentally measured $\Upsilon(1 S)$ mass and comparing the subtracted

\footnotetext{
${ }^{2}$ The perturbative ultrasoft correction itself starts at $O\left(m \alpha_{s}^{5}\right)[37$.
} 
mass with the PMS value of the resummed energy, $2 m_{\mathrm{BR}}+E_{\mathrm{BR}}$, we obtain $m_{\mathrm{BR}}=4.89 \pm 0.03$ $\mathrm{GeV}$.

For the error estimate we notice in Fig. 4 the resummed energies at NLO, NNLO, and NNNLO converge around $\mu \approx 2.5 \mathrm{GeV}$ and the PMS scales for the NNLO and NNNLO resummed energies lie within the range $m_{\mathrm{Bohr}} \leq \mu \leq 5 \mathrm{GeV}$, where $m_{\mathrm{Bohr}} \approx 2 \mathrm{GeV}$. We therefore take the variations of the resummed energies in this range as the error estimate. Then the uncertainties in the $a_{3}$ and the strong coupling give $\pm 27 \mathrm{MeV}$ and $\pm 13 \mathrm{MeV}$, respectively, to the binding energy, and the renormalization scale dependence and the uncertainty in the residue (20) cause another $\pm 30 \mathrm{MeV}$ and $\pm 12 \mathrm{MeV}$, respectively. The error due to the unknown higher order contribution should be partly covered by the error from the residue. However, setting this fact aside we shall assign an independent error $\pm 35 \mathrm{MeV}$ to the binding energy, which is the difference between the respective energies at NNLO and at NNNLO at the Bohr scale. It is interesting to observe that the PMS values at NNLO and NNNLO are virtually identical, an indication that the unknown higher order effect should be small.

Combining these errors (including those from the gluon condensate and the nonzero charm mass effect) in quadrature we have the BR mass for the bottom quark

$$
m_{\mathrm{BR}}^{(b)}=4.89 \pm 0.04 \mathrm{GeV} .
$$

Now, from the BR mass the $\overline{\mathrm{MS}}$ mass can be obtained through the relation, $m_{\overline{\mathrm{MS}}}^{(b)}\{1+$ $\mathcal{M}_{\mathrm{BR}}\left[\alpha_{s}^{[4]}\left(m_{\frac{(b)}{\mathrm{MS}}}^{(b)}\right]\right\}=m_{\mathrm{BR}}^{(b)}$, from which we obtain

$$
m \frac{(b)}{\mathrm{MS}}=4.20 \pm 0.04 \mathrm{GeV} .
$$

Compare this to the result from Ref. [12],

$$
m \frac{(b)}{\mathrm{MS}}=4.346 \pm 0.070 \mathrm{GeV},
$$

which was obtained without taking the renormalon into account. Notice that the central values have a significant difference, and our value has a smaller error. The reduction in the error largely comes from the smaller renormalization scale dependence. The assigned error for the scale dependence in (38) is more than four times larger than ours, because of much stronger scale dependence in its derivation. This comparison shows that renormalon effect is significant and must be taken into account for accurate determination of the bottom quark mass. Our value for the bottom quark mass (37) compares favorably with the values collected in [41].

\section{DISCUSSION}

In this paper we have shown that an accurate calculation of the normalization constant of the large order behavior and information on the renormalon singularity allows us a precision calculation of the pole mass and quarkonium binding energy. The existing method of handling the renormalon problem was bypassing it, by concentrating exclusively on directly measurable observables and employing renormalon-free short distance masses. In contrast, in our approach each of the first IR renormalon in IR sensitive quantities is resummed independently, and as a consequence those IR sensitive quantities can be extracted accurately from experimental data. 
One of the advantages of our approach is that it provides a natural solution to the scale mixing problem of the approaches based on renormalon cancellation. In systems with farseparated multiple scales the renormalon cancellation approach inevitably mixes those scales through the renormalization scale, forcing one to choose an optimal scale over a wide range of scales. Depending on the problem this can be a source of significant error. For instance, in the top-pair threshold production the normalization of the production cross section is known to be sensitive to the choice of the renormalization scale [42], which can limit the accuracy of the extracted strong coupling constant. With our approach, this problem can be solved by resumming the top pole mass and the interquark potential at their respective optimal scales, namely, the top mass and the interquark distance.

This suggests a potential improvement in the bottom quark mass determination. Instead of applying the resummation to the perturbatively calculated binding energy, as we did in this paper, one may solve the Schroedinger equation, which was advocated in [43], with a resummed interquark potential to obtain a more accurate binding energy. It should be noted, however, presently the error in the bottom quark mass is dominated by the nonperturbative effect.

\section{Acknowledgments}

The author wish to thank A. Pineda for patiently answering questions. He also thanks Kingman Cheung and high energy theory group at NCTS, Hsinchu, Taiwan, for warm hospitality extended to the author during his visit. This work was supported partly by the BK21 program.

[1] I. I. Y. Bigi, M. A. Shifman, N. G. Uraltsev, and A. I. Vainshtein, The pole mass of the heavy quark. perturbation theory and beyond, Phys. Rev. D50 (1994) 2234-2246, hep-ph/9402360.

[2] M. Beneke and V. M. Braun, Heavy quark effective theory beyond perturbation theory: Renormalons, the pole mass and the residual mass term, Nucl. Phys. B426 (1994) 301-343, hep-ph/9402364.

[3] M. Beneke, More on ambiguities in the pole mass, Phys. Lett. B344 (1995) 341-347, hep-ph/9408380.

[4] M. Beneke, V. M. Braun, and V. I. Zakharov, Bloch-nordsieck cancellations beyond logarithms in heavy particle decays, Phys. Rev. Lett. 73 (1994) 3058-3061, hep-ph/9405304.

[5] M. Neubert and C. T. Sachrajda, Cancellation of renormalon ambiguities in the heavy quark effective theory, Nucl. Phys. B438 (1995) 235-260, hep-ph/9407394.

[6] M. E. Luke, A. V. Manohar, and M. J. Savage, Renormalons in effective field theories, Phys. Rev. D51 (1995) 4924-4933, hep-ph/9407407.

[7] T. Lee, Renormalons beyond one-loop, Phys. Rev. D56 (1997) 1091-1100, hep-th/9611010.

[8] T. Lee, Normalization constants of large order behavior, Phys. Lett. B462 (1999) 1-6, hep-ph/9908225.

[9] T. Lee, Surviving the renormalon in heavy quark potential, Phys. Rev. D67 (2003) 014020, hep-ph/0210032.

[10] T. Lee, Direct resummation of the leading renormalons in inclusive semileptonic $b$ decay, Phys. Lett. B563 (2003) 93, hep-ph/0212034. 
[11] A. Pineda, Determination of the bottom quark mass from the upsilon(1s) system, JHEP 06 (2001) 022, hep-ph/0105008.

[12] A. A. Penin and M. Steinhauser, Heavy quarkonium spectrum at o(alpha $(s) * * 5 m(q))$ and bottom / top quark mass determination, Phys. Lett. B538 (2002) 335-345, hep-ph/0204290.

[13] N. Gray, D. J. Broadhurst, W. Grafe, and K. Schilcher, Three loop relation of quark (modified) ms and pole masses, Z. Phys. C48 (1990) 673-680.

[14] K. Melnikov and T. v. Ritbergen, The three-loop relation between the ms-bar and the pole quark masses, Phys. Lett. B482 (2000) 99-108, hep-ph/9912391.

[15] K. G. Chetyrkin and M. Steinhauser, The relation between the ms-bar and the on-shell quark mass at order alpha(s)**3, Nucl. Phys. B573 (2000) 617-651, hep-ph/9911434.

[16] K. S. Jeong and T. Lee, Estimating higher order perturbative coefficients using borel transform, Phys. Lett. B550 (2002) 166-171, hep-ph/0204150.

[17] G. Cvetic and T. Lee, Bilocal expansion of borel amplitude and hadronic tau decay width, Phys. Rev. D64 (2001) 014030, hep-ph/0101297.

[18] S. Titard and F. J. Yndurain, Rigorous qcd evaluation of spectrum and ground state properties of heavy $q$ anti- $q$ systems: With a precision determination of $m(b) m($ eta $(b))$, Phys. Rev. D49 (1994) 6007-6025, hep-ph/9310236.

[19] S. Titard and F. J. Yndurain, Rigorous qcd evaluation of spectrum and other properties of heavy $q$ anti-q systems. 2. bottomium with $n=2, l=0$, 1, Phys. Rev. D51 (1995) 6348-6363, hep-ph/9403400.

[20] Y. Kiyo and Y. Sumino, $O\left(\operatorname{alpha}(\mathrm{s})^{* * 5} \mathrm{~m}\right)$ quarkonium $1 \mathrm{~s}$ spectrum in large beta $(0)$ approximation and renormalon cancellation, Phys. Lett. B496 (2000) 83-88, hep-ph/0007251.

[21] Y. Kiyo and Y. Sumino, Top mass determination and o(alpha $\left.(\mathrm{s})^{* * 5} \mathrm{~m}\right)$ correction to toponium 1 s energy level, Phys. Rev. D67 (2003) 071501, hep-ph/0211299.

[22] A. Pineda and F. J. Yndurain, Calculation of quarkonium spectrum and $m(b), m(c)$ to order alpha(s)**4, Phys. Rev. D58 (1998) 094022, hep-ph/9711287.

[23] B. A. Kniehl and A. A. Penin, Order alpha(s)**3 ln**2(1/alpha(s)) corrections to heavyquarkonium creation and annihilation, Nucl. Phys. B577 (2000) 197-208, hep-ph/9911414.

[24] N. Brambilla, A. Pineda, J. Soto, and A. Vairo, The heavy quarkonium spectrum at order $m$ alpha(s)**5 ln(alpha(s)), Phys. Lett. B470 (1999) 215, hep-ph/9910238.

[25] B. A. Kniehl, A. A. Penin, V. A. Smirnov, and M. Steinhauser, Potential nrqcd and heavy-quarkonium spectrum at next-to- next-to-next-to-leading order, Nucl. Phys. B635 (2002) 357-383, hep-ph/0203166.

[26] F. A. Chishtie and V. Elias, Rg/pade estimate of the three-loop contribution to the qcd static potential function, Phys. Lett. B521 (2001) 434-440, hep-ph/0107052.

[27] A. H. Hoang, M. C. Smith, T. Stelzer, and S. Willenbrock, Quarkonia and the pole mass, Phys. Rev. D59 (1999) 114014, hep-ph/9804227.

[28] M. Beneke, A quark mass definition adequate for threshold problems, Phys. Lett. B434 (1998) 115-125, hep-ph/9804241.

[29] S. Recksiegel and Y. Sumino, Improved perturbative qcd prediction of the bottomonium spectrum, Phys. Rev. D67 (2003) 014004, hep-ph/0207005.

[30] Particle Data Group Collaboration, K. Hagiwara et. al., Review of particle physics, Phys. Rev. D66 (2002) 010001.

[31] P. M. Stevenson, Optimized perturbation theory, Phys. Rev. D23 (1981) 2916. 
[32] K. G. Chetyrkin, J. H. Kuhn, and M. Steinhauser, Rundec: A mathematica package for running and decoupling of the strong coupling and quark masses, Comput. Phys. Commun. 133 (2000) 43-65, hep-ph/0004189.

[33] M. B. Voloshin, On dynamics of heavy quarks in nonperturbative qcd vacuum, Nucl. Phys. B154 (1979) 365.

[34] H. Leutwyler, How to use heavy quarks to probe the qcd vacuum, Phys. Lett. B98 (1981) 447.

[35] B. V. Geshkenbein, B. L. Ioffe, and K. N. Zyablyuk, The check of qcd based on the tau decay

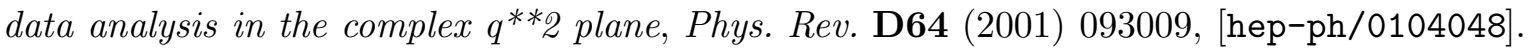

[36] M. A. Shifman, A. I. Vainshtein, and V. I. Zakharov, Qcd and resonance physics. sum rules, Nucl. Phys. B147 (1979) 385-447.

[37] B. A. Kniehl and A. A. Penin, Ultrasoft effects in heavy quarkonium physics, Nucl. Phys. B563 (1999) 200-210, hep-ph/9907489.

[38] D. Gromes, Space-time dependence of the gluon condensate correlation function and quarkonium spectra, Phys. Lett. B115 (1982) 482.

[39] A. H. Hoang, Bottom quark mass from upsilon mesons: Charm mass effects, hep-ph/0008102.

[40] D. Eiras and J. Soto, Light fermion finite mass effects in non-relativistic bound states, Phys. Lett. B491 (2000) 101-110, hep-ph/0005066.

[41] A. X. El-Khadra and M. Luke, The mass of the b quark, Ann. Rev. Nucl. Part. Sci. 52 (2002) 201-251, hep-ph/0208114.

[42] A. H. Hoang et. al., Top-antitop pair production close to threshold: Synopsis of recent nnlo results, Eur. Phys. J. direct C2 (2000) 3, hep-ph/0001286.

[43] W. Lucha and F. F. Schoberl, Improved mass of the bottom quark, Phys. Rev. D62 (2000) 097501. 